\title{
SULL'ORIGINE DEL ROMBO DEL TUONO
}

\author{
Guglielmo Zanotelli
}

Sulle probabili cause del rombo prolungato che spesso si accompagna allo scoppio del tuono non si rinvengono consistenti elementi nella moderna letteratura scientifica; le idee in proposito non hanno subito sensibile evoluzione rispetto a quelle che, in base alla semplice osservazione del fenomeno naturale, avevano potuto farsene gli antichi autori. Humphreys nel suo trattato sulla fisica dell'atmosfera (') ascrive a tre diversi ordini di fatti il nascere del rombo del tuono, e cioè la lunghezza e la tortuosità del cammino del fulmine, il che porta in istanti differenti la percezione sonora all'orecchio dell'osservatore, il ripetersi delle scariche, ed infine la riflessione causata dagli oggetti circostanti, come le montagne e le colline: aggiunge lo stesso autore che "l'importanza di questo ultimo fattore è generalmente sopravalutata, giacché il rombo del tuono è ordinariamente il medesimo sia sull'oceano che su una prateria od in mezzo alle montagne ". Alla lunga durata ed alle variazioni di intensità che caratterizzano il rombo del tuono non sono certamente estranee le prime due cause, ed anche la inomogeneità dell'atmosfera, per la parte interessata al fenomeno, come aveva già ritenuto Laska $\left({ }^{2}\right)$ : anzi a tale inomogeneità può ascriversi, come ha mostrato recentemente Fleagle $\left({ }^{3}\right)$, la limitazione dè raggio di udibilità al crescere della distanza dell'osservatore dalla sede della scarica elettrica. Ma il terzo fattore, cioè la riflessione, è senza dubbio da ritenersi di rilevante importanza, se ci permetterà, come mostreremo in questo lavoro, di dar conto direttamente di due delle più singolari caratteristiche del tuono, cioè la notevole persistenza ed il peculiare timbro, eminentemente ricco di suoni bassi in confronto al colpo secco e chiaro del fulmine caduto in immediata prossimità dell'osservatore.

Nella antica memoria di Arago ( $\left.{ }^{4}\right)$ dedicata al tuono ed al fulmine si notano fra l'altro alcune osservazioni in cui è contenuta, in germe, la spiegazione che noi forniremo del fenomeno che ci interessa. Dice infatti Arago: "qualche volta il rumore del tuono sembra chiaro e secco, come quello di un semplice colpo di pistola; più generalmente 
è però pieno e molto grave. Alcuni osservatori pretendono perfino che diventi sempre più grave via via che il rimbombo si prolunga ». E più oltre aggiunge: " $i$ marinai assicurano che in mare aperto il fulmine è accompagnato da lunghi rimbombi come a terra, quantunque non ci siano, per riflettere il suono, né mura, né rocce, né boschi, né colline, né montagne. Coloro che si fondano su questa enumerazione dimenticano le nubi, o piuttosto ammettono che le nubi non godano della proprietà di riflettere il suono $)$.

Il fatto che gli ammassi di goccioline di acqua che costituiscono le nubi possano esercitare un'azione rilevante sulla propagazione dei suoni, azione che si estrinseca sostanzialmente come un assorbimento selettivo per certe frequenze, risulta da due lavori recenti: l'uno di Oswatitsch $\left({ }^{5}\right)$ che studiò il fenomeno termodinamico della alterna condensazione ed evaporazione delle goccioline per effetto delle variazioni della pressione acustica; l'altro dello scrivente $\left({ }^{6}\right)$, che si occupò del fenomeno meccanico del trascinamento delle goccioline da parte della oscillazione sonora. Ambedue i fenomeni danno origine, sia pure in campi diversi di frequenze, a dissipazione di energia sonora in corrispondenza alle frequenze più alte; anzi Oswatitsch aveva già intuitivamente ascritto al fattore termodinamico il forte assorbimento del tuono da parte delle formazioni nuvolose. Tuttavia a questo effetto cooperano evidentemente ambedue $i$ fattori: solo uno studio circostanziato delle modalità del fenomeno potrà permetterci di esaminarne la rispettiva importanza.

Il prevalente assorbimento di energia sonora del tuono da parte delle nubi nella gamma delle frequenze acustiche elevate può derivare dall'andamento del coefficiente di assorbimento specialmente per la parte inerente al fenomeno meccanico, coefficiente di assorbimento che cresce, come mostrai nel già citato lavoro, in modo rilevante al crescere della frequenza. Tuttavia se questa interpretazione è valida a spiegare il timbro cupo del rumore del fulmine che scoppia nell'interno di una formazione nuvolosa, non si applica per i fulmini che avvengono esternamente, specialmente per quelli che si scaricano al suolo, e lascia inoltre impregiudicata la questione della persistenza del rombo del tuono. Senza volere escludere l'influenza del fattore assorbimento, mi occuperò in particolare nel presente lavoro del caso che lo scoppio del fulmine sia esterno alla formazione nuvolosa, nella quale eventualità gli effetti acustici sono da imputarsi in gran parte alla riflessione. 
Mi propongo di mostrare come un banco di nubi, che supporrò per semplicità di grande spessore, lateralmente indefinito, delimitato verso la libera atmosfera da una superficie piana, e composto di goccioline di acqua sferiche, tutte uguali ed uniformemente distribuite, si comporta per l'onda sonora che l'investe sotto incidenza normale come un filtro passa basso per riflessione: nel senso cioè che ne vengono riflesse con uguale attenuazione tutte le frequenze sino ad una certa frequenza di taglio, oltre la quale rapidamente il potere riflettente si perde.

Non ho preso in considerazione lo spessore del banco nuvoloso, supponendolo sempre sufficientemente grande da poter trascurare gli effetti del passaggio dell'onda sonora attraverso alla seconda superficie di delimitazione con l'atmosfera; in questo stesso senso parlerò nel seguito anche di strato nuvoloso, limitandomi sempre a considerare la riflessione attraverso la sola superficie d'ingresso: perché questa condizione sia effettivamente soddisfatta è sufficiente per lo più ammettere uno spessore relativamente esiguo dello strato, per il fatto che il coefficiente di assorbimento per la propagazione dell'onda sonora nell'interno delle nubi è in genere relativamente elevato, specie per le frequenze più alte $\left({ }^{7}\right.$ ).

Lo strato nuvoloso opera una selezione tra le componenti armoniche della perturbazione acustica generata dallo scoppio del fulmine, riflettendo solo quelle di frequenza più bassa ed assorbendo le restanti. La parte riflessa poi può essere soggetta ad ulteriori riflessioni, sia perché in realtà le nubi non sono distribuite in un unico strato, sia per la presenza del suolo e degli ostacoli terrestri. Tali riflessioni successive esaltano sempre più le basse frequenze e prolungano la durata del fenomeno, dando ragione del cupo e prolungato rimbombo che rende così caratteristico lo scoppio del tuono. Ciò non vuol dire naturalmente che altre circostanze non possano cooperare a questo effetto, come quelle già indicate, e cioè il susseguirsi delle scariche elettriche e la lunghezza e tortuosità del cammino del fulmine.

La grandezza del coefficiente di riflessione ed il valore della frequenza di taglio dipendono, come vedremo, dalle dimensioni e dalla quantità delle goccioline contenute per unità di volume di nube, oltre che dalla natura del fenomeno interessato, cioè se meccanico o termodinamico. Il coefficiente di riflessione per condensazione risulta in genere maggiore: tuttavia essendo la relativa frequenza di taglio più 
bassa può accadere, per nubi composte di goccie molto fitte e minute, che questa cada addirittura al disotto o attorno al limite inferiore di udibilità. In tal caso della riflessione è responsabile il solo fattore meccanico, cui corrisponde un coefficiente di assorbimento assai minore, ma una frequenza di taglio più elevata. A compensare almeno in parte la esiguità del coefficiente di riflessione per effetto meccanico interviene però la maggior sensibilità dell'orecchio umano alle frequenze più alte.

Per determinare i coefficienti di riflessione conviene riannodarsi ai risultati delle due trattazioni, termodinamica e meccanica, sul passaggio di un'onda sonora piana di frequenza $\omega / 2 \pi$ attraverso allo strato nuvoloso.

Nella prima trattazione si considera ciascuna delle goccioline uguali di raggio a contenute nell'unità di volume di aria come assoggettata ad un alterno processo di evaporazione e di condensazione

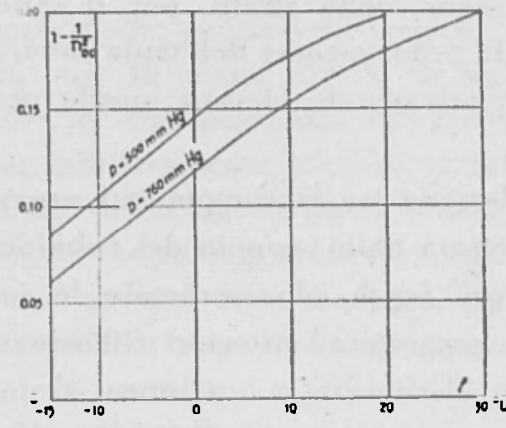

Fig. 1 sotto l'azione delle periodiche variazioni della pressione acustica. Fra i due casi estremi, che la frequenza del suono sia cosi bassa in confronto della velocità di condensazione del vapor d'acqua sulle goccie che in ogni istante si possa raggiungere l'equilibrio di saturazione (propagazione del suono in regime di adiabatica umida), oppure cosi alta che praticamente la condensazione venga a mancare e la propagazione si svolga come in assenza delle goccie (regime di adiabatica asciuttal, esiste un campo di frequenze intermedio in cui si verifica dispersione, cioè dipendenza della velocità di propagazione dell'onda sonora della frequenza, insieme ad assorbimento, cioè trasformazione dell'energia acustica in calore. In questo campo intermedio vi è dunque luogo alla considerazione di un indice di rifrazione complesso

$$
n_{\mathrm{c}}=1+v_{\mathrm{c}}-j u_{\mathrm{c}}
$$

relativo all'aria secca in assenza di goccie, che, secondo $i$ risultati del calcolo di Oswatitsch, è dato (") dalla relazione

* Ho alquanto modificato le notazioni di Oswatitsch, per accordarle il meglio possibile con quelle del mio precedente lavoro. 


$$
1-\frac{1}{n^{2}{ }_{\mathrm{c}}}=\frac{1-\frac{1}{n^{2}{ }_{\mathrm{oc}}}}{1-\left(\frac{\omega}{(1)_{\mathrm{c}}}\right) g+j \frac{\omega}{\omega_{\mathrm{c}}}},
$$

ove $n_{o c}$ è l'indice di rifrazione per la frequenza zero, che risulta reale: è quest'ultimo l'indice di rifrazione per la propagazione in aria satura di umidità, cioè quello calcolabile in base all'equazione di propaga-

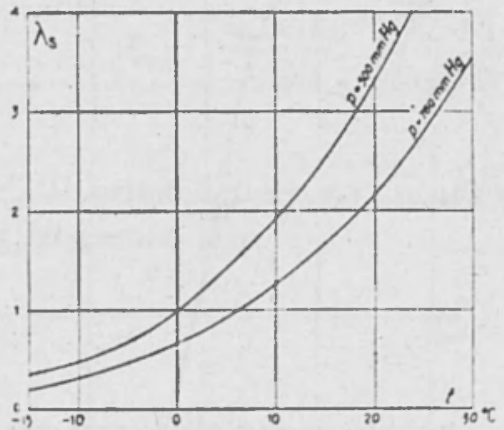

Fig. 2

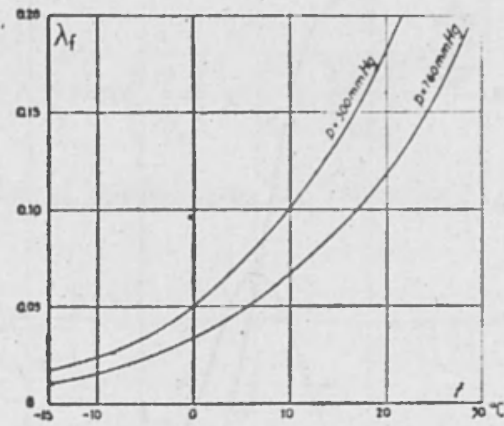

Fig. 3

zione del suono a partire dalla adiabatica umida: il grafico di fig. 1 , riprodotto dal lavoro di Oswatitsch, riporta appunto l'andamento di $1-1 / n^{2}{ }_{\text {oce }}$ Inoltre $\omega_{\mathrm{c}}$ e $g$ sono due parametri caratteristici dati da

$$
\begin{aligned}
& \omega_{\mathrm{c}}=\frac{a}{x^{2}} \frac{3 D\left[1+\gamma\left(\lambda_{0}-\lambda_{\mathrm{f}}\right)\right]}{1+\frac{a_{\mathrm{o}}}{a}+\frac{\lambda_{\mathrm{e}} c_{\mathrm{p}} \mathrm{Q} D}{k}} \\
& g=q\left(1+\frac{a_{\mathrm{o}}}{a}\right) \frac{c_{1} \varrho_{1} D\left[1+\gamma\left(\lambda_{0}-\lambda_{\mathrm{f}}\right)\right]}{k\left(1+\frac{a_{\mathrm{o}}}{\boldsymbol{a}}+\frac{\lambda_{\mathrm{s}} c_{\mathrm{p}}{ } D}{k}\right)}=.
\end{aligned}
$$

In queste formule $\varrho, k, c_{\mathrm{pu}} \gamma$, rappresentano rispettivamente la masza specifica, il coefficiente di conducibilità termica, il calore specifico a pressione costante, ed il rapporto di quest'ultimo al calore specifico a volume costante per l'aria; $\varrho_{1}, c_{1}$ la massa specifica ed il calore specifico dell'acqua; $D$ il coefficiente di diffusione del vapore acqueo nell'aria; $a$ e $a_{0}$ il raggio delle goccioline di acqua costituenti la nube e rispettivamente un raggio caratteristico nel processo di accrescimento delle goccioline stesse per condensazione, che, secondo Oswatitsch, può prevedersi per le normali circostanze meteorologiche alle nostre latitudini essere $a_{0}=5.10^{-1} \mathrm{~cm} ; q$ il rapporto fra $i$ volumi 
occupati nella nube dall'acqua contenutavi sotto forma di goccie e rispettivamente dall'aria. Infine $\lambda_{\mathrm{f}}$ e $\lambda_{\mathrm{f}}$ sono due funzioni della temperatura e della pressione rappresentate nei grafici di figure 2 e 3 , riprodotti dal lavoro di Oswatitsch.

Dei due parametri $\omega_{c}$ e $g$, il primo risulta avere le dimensioni di una pulsazione, ed individua il centro della banda di dispersione. Il numero puro $g$ che è proporzionale al rapporto $q$ ha invece scarsa

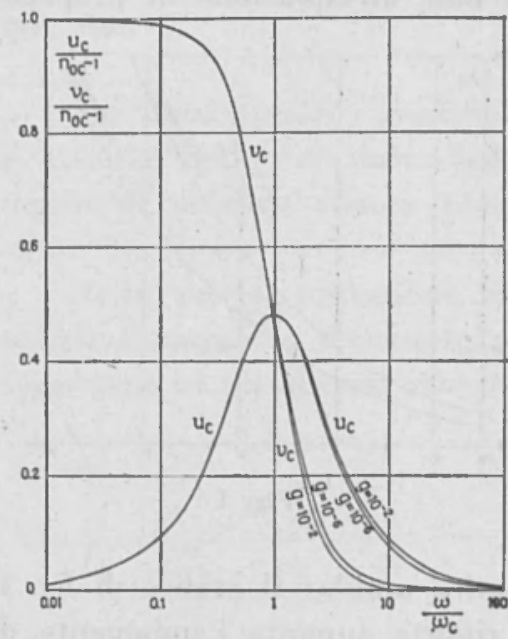

Fig. 4

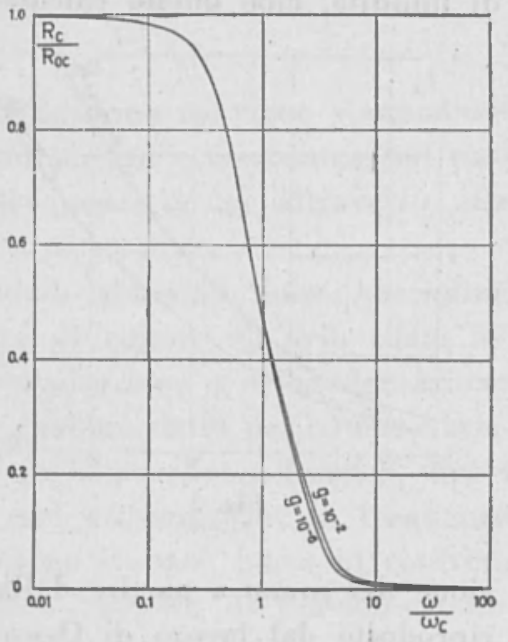

Fig. 5

importanza pratica nelle ordinarie condizioni meteorologiche; il suo valore si mantiene sempre piuttosto piccolo: anche assumendo $q=10^{-5}$, corrispondente al forte quantitativo di $10 \mathrm{~g}$ di acqua per $\mathrm{m}^{3} \mathrm{di}$ nube, si hanno al massimo per $g$ valori dell'ordine di $10^{-2}$.

Nel grafico di fig. 4 è riportato l'andamento dell'eccedenza della parte reale dall'unità, e del coefficiente della parte immaginaria dell'indice di rifrazione $n_{c}$ in funzione del rapporto $\omega / \omega_{c}$, dal quale risulta appunto la scarsa influenza del valore di $g$; le curve sono state costruite in base alla [2] assumendo per la frequenza zero il valore $n_{\mathrm{oc}}=1,1$, corrispondente, secondo il grafico di fig. 1 , ad una pressione atmosferica di $600 \mathrm{mmHg}$ e ad una temperatura di $10^{\circ} \mathrm{C}$.

Il coefficiente di riflessione, cioè il rapporto fra l'intensità del raggio sonoro riflesso e l'intensità del raggio incidente normalmente sullo strato nuvoloso, è dato per il caso delle onde piane da

$$
R_{\mathrm{e}}=\left|\frac{n_{\mathrm{e}}-1}{n_{\mathrm{c}}+1}\right|^{2},
$$


e poiche $\left|n_{c}\right|$ è sempre poco diverso dall'unità, il valore ne è sostanzialmente determinato da quello di $\left|n_{c}-1\right|^{2}$. La [2] può mettersi nella forma

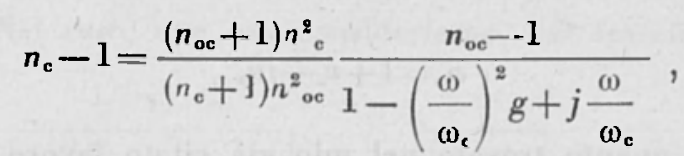

e quindi abbiamo

$$
R_{\mathrm{c}}=\left|\frac{n_{\mathrm{oc}}-1}{n_{\mathrm{oc}}+1}\right|^{2}\left|\frac{\left(n_{\mathrm{oc}}+1\right) n_{\mathrm{c}}}{\left(n_{\mathrm{c}}+1\right) n_{\mathrm{o}}}\right|^{4} \frac{1}{\left[1-\left(\frac{\omega}{\omega_{\mathrm{c}}}\right)^{2} g\right]^{2}+\left[\frac{(1)}{\omega_{\mathrm{c}}}\right]^{2}} \cdot[7]
$$

Il primo fattore non è altro che il coefficiente di riflessione per la frequenza zero

$$
R_{\mathrm{ne}}=\left|\frac{n_{\mathrm{oc}}--1}{n_{\mathrm{oc}}+1}\right|^{2}
$$

e per il caso da noi considerato che sia $n_{o c}=1,1$, si ha che $R_{\text {oc }}$ è circa $2,3 \cdot 10^{-3}$. Il secondo fattore

$$
K(\omega)=\left|\frac{\left(n_{\mathrm{oc}}+1\right) n_{\mathrm{c}}}{\left(n_{\mathrm{c}}+1\right) n_{\mathrm{oc}}}\right|^{4}
$$

varia poco con la frequenza e si mantiene sempre prossimo all'unità : è $K(0)=1$ e $K(\infty)=0,8$. Il terzo fattore infine ha lo stesso andamento di $\left|n_{c}-1\right|^{2}=u^{2}+v^{2}{ }_{c}$, e come questa grandezza viene a dipendere poco da $g$, in quanto il termine che contiene la $g$ prende rilievo nel denominatore della [6] solo per valori $\mathrm{di} \omega / \omega_{\mathrm{c}}$ molto grandi, cioè dove $R_{c}$ è già raolto piccolo.

Il coefficiente di riflessione si mantiene all'incirca uguale a quello per la frequenza zero sinché è $\omega / \omega_{c}<<1$, comincia a diminuire allorché $\omega / \omega_{\mathrm{c}}$ è prossimo all'unità, per cadere poi rapidamente quando $\omega / \omega_{c}$ prende valori maggiori. Possiamo assumere come frequenza di taglio proprio la $\omega_{\mathrm{c}} / 2 \tau$, al quale valore il coefficiente di riflessione si è ridotto a circa $0,5 R_{o c}$. Il grafico di fig. 5 riporta l'andamento del coefficiente di riflessione per il caso già da noi considerato di una pressione di $600 \mathrm{mmHg}^{\mathrm{ed}}$ una temperatura di $10^{\circ} \mathrm{C}$.

Consideriamo ora invece il fenomeno della dispersione e dell'assorbimento del suono da parte del solito banco nuvoloso, per effetto 
dell'attrito opposto dalla viscosità dell'aria al moto cui le goccioline d'acqua sono sollecitate dall'onda sonora. Anche qui ha luogo la considerazione di un indice di rifrazione complesso

$$
n_{*}=1+v_{*}-j u_{*},
$$

che, secondo quanto trovato nel mio già citato lavoro, è dato dalla relazione

$$
n_{2}-1=\frac{1}{2} q(\varepsilon-1)\left\{1-\frac{\frac{\varepsilon}{\varepsilon-1}}{1+\frac{3}{2 \varepsilon}+\frac{0}{4 \varepsilon z}-j \frac{0}{4 \varepsilon z}\left(1+\frac{1}{z}\right)}\right\} \text {, }
$$

dove i paramenti $\varepsilon$ e $z$ valgono

$$
\varepsilon=\frac{Q_{1}}{\varrho}-1 \quad, \quad z=a \sqrt{\frac{\omega}{\bar{z} v}},
$$

essendo $v$ il coefficiente di viscosità cinematico dell'aria, ossia il rapporto dell'ordinario coefficiente di viscosità alla massa specifica. Dalla precedente appare chiaro che alla frequenza zero l'indice di rifrazione $n$, è reale e dato da

$$
n_{\mathrm{ou}}-1=\frac{1}{2} q(\varepsilon-1) .
$$

Se anche si prende il valore assai forte $q=10^{-\Sigma}$, essendo $\varepsilon$ circa $10^{3}$ per le solite condizioni di temperatura e di pressione, risulta $n_{\mathrm{os}}-\mathrm{l}=5.10^{-3}$, cioè l'indice di rifrazione solơ di pochissimo maggiore dell'unità. Il grafico di figura 6 riporta l'andamento dell'eccedenza della parte reale dall'unità, e del coefficiente di quella immaginaria dell'indice di rifrazione $n$, in funzione della varialile $z$. L'indice di rifrazione si comporta, almeno qualitativamente, come nel caso precedentemente considerato, per quanto il fatto fisico ne sia sostanzial. mente differente. Ciò non reca meraviglia se si riflette che $i$ due fenomeni dell'alterna condensazione ed evaporazione delle goccie, e rispettivamente del trascinamento delle stesse nel moto dell'onda sono. ra si presentano con modalità affini: cioè si ha una banda delle frequenze basse in cui il fenomeno fisico è in grado di seguire fedel. 
mente le variazioni dell'onda sonora; una banda delle alte frequenze in cui invece prevale l'inerzia ed il fenomeno non si verifica più che in misura irrilevante; infine una banda intermedia ove si presenta la dispersione. Nel caso, che ora consideriamo, del trascinamento mec-

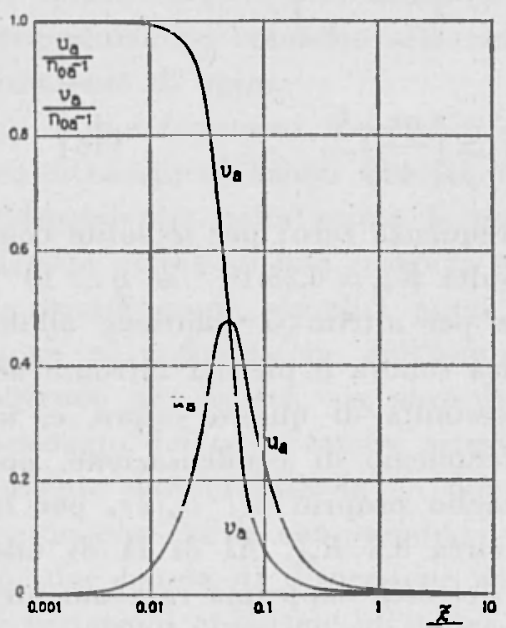

Fig. 6

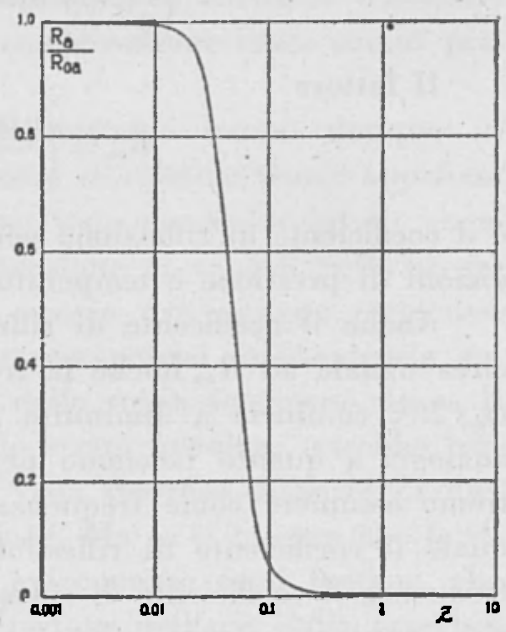

Fig. 7

canico il centro della banda di dispersione corrisponde all'incirca al valore

$$
z=\frac{3}{2 \sqrt{\varepsilon}}
$$

cioè ad una pulsazione

$$
\omega_{\mathrm{a}}=\frac{1}{a^{\circ}} \frac{9 v}{2 \varepsilon} .
$$

Dalla espressione [11] dell'indice di rifrazione siamo ora in grado di determinare il coefficiente di riflessione relativo al fenomeno di attrito

$$
R_{\mathrm{a}}=\left|\frac{n_{\mathrm{a}}-1}{n_{\mathrm{a}}+1}\right|^{2} \cong \frac{1}{4}\left|n_{\mathrm{a}}-1\right|^{\mathrm{2}}
$$

Infatti nel caso presente essendo $|\boldsymbol{n}|$ sempre vicinissimo all'unità, può a denominatore sostituirsi al posto di $|n+1|^{2}$ addirittura 4. Il cal- 
colo del coefficiente di riflessione a partire dalla [11] conduce alla seguente espressione

$$
R_{\mathrm{a}}=\left(\frac{q(\varepsilon-1)}{4}\right)^{2} \frac{\left(\frac{2(\varepsilon-3)}{1(z-1)} z+1\right)^{2}+\left(1+\frac{1}{z}\right)^{2}}{\left(\frac{2}{9}(2 \varepsilon+3) z+1\right)^{2}+\left(1+\frac{1}{z}\right)^{2}} .
$$

Il fattore

$$
R_{\mathrm{oa}}=\left(\frac{q(\varepsilon-1)}{4}\right)^{2} \cong\left(\frac{q \varepsilon}{4}\right)^{2}
$$

è il coefficiente di riflessione per la frequenza zero; per le solite condizioni di pressione e temperatura risulta $R_{\text {os }}=6,25 \cdot 10^{-6}$ se $q=10^{-5}$.

Anche il coefficente di riflessione per attrito si mantiene all'incirca uguale ad $R_{\text {on }}$ finché la frequenza sonora è piccola difronte ad $\omega_{\mathrm{a}} / 2 \pi$; comincia a diminuire in prossimità di questo valore, $e$, in analogia a quanto facemmo per il fenomeno di condensazione, potremo assumere come frequenza di taglio proprio la $\omega_{\mathrm{a}} / 2 \pi$, per la quale il coefficiente di riflessione è circa $0,5 R_{\text {ua }}$. Al di là di tale frequenza il coefficiente di riflessione decresce dapprima rapidamente, poi sempre più lentamente sino a tendere, per $z>>1$, al valore costante e piccolissimo

$$
\left(\frac{q}{4} \frac{\varepsilon-3}{2 \varepsilon+3}\right)^{2}=\left(\frac{q}{8}\right)^{2}
$$

Questo andamento del coefficiente di riflessione per attrito in funzione di $z$ risulta chiaramente dal grafico di fig. 7 .

Il rapporto fra le frequenze di taglio [3] e [15] per condensazione e per attrito

$$
\frac{\omega_{\mathrm{c}}}{\omega_{\mathrm{s}}}-\frac{2 \varepsilon D}{3 v} \frac{1+\gamma\left(\lambda_{\mathrm{s}}-\lambda_{\mathrm{f}}\right)}{1+\frac{a_{\mathrm{o}}}{a}+\frac{\lambda_{\mathrm{s}} c_{\mathrm{p}} \varrho D}{k}},
$$

è sempre notevolmente minore dell'unità e cresce al crescere di $q \mathrm{e}$ di $a$ sino a raggiungere la grandezza di circa $10^{-2}$ per i valori massimi ammissibili in pratica $q=10^{-5}$ ed $a=10^{-2} \mathrm{~cm}$. La frequenza $\omega_{\pi} / 2 \pi$ per attrito $\grave{e}$ dunque sempre almeno un centinaio di volte maggiore di quella $\omega_{\mathrm{c}} / 2 . \tau$ per condensazione. $\mathrm{Si}$ verifica quindi subito per mezzo delle [6] e [11], se ci riferiamo per esempio alle già considerate condizioni di temperatura e di pressione, che $\left|n_{\mathrm{c}}-1\right| \mathrm{j}$ è una trentina di volte più grande di $\left|n_{a}-1\right|$ in corrispondenza ad $\omega=\omega_{c}$, 
mentre per $\omega=\omega_{\mathrm{a}} \grave{\mathrm{e}}\left|n_{\mathrm{g}}-1\right|$ già divenuto più di tre volte maggiore di $\left|n_{\mathrm{c}} \quad 1\right|$. Dunque le due bande di dispersione per condensazione e rispettivamente per attrito sono situate così lontane luna dall'altra cbe in corrispondenza a quella di condensazione il fenomeno meccanico dell'attrito è in confronto ancora irrilevante, ed invece in corrispondenza alla banda di dispersione per attrito il fenomeno termodinamico connesso alla alterna condensazione cessa ormai praticamente di agire.

I due fenomeni meccanico e termodinamico hanno dunque in realtà campi di azioni distinti, così come le relative teorie appaiono indipendenti: nella prima le goccioline sono concepite infatti come sferette mobili di una sostanza senza tensione di vapore, nella seconda invece come semplici punti dello spazio ove avviene condensazione ed evaporazione dell'acqua. Tuttavia poiché queste teorie descrivono due aspetti, sia pure diversi, dello stesso fenomeno fisico, il passaggio dell'onda sonora attraverso lo strato nuvoloso, sarebbe certamente desiderabile da un punto di vista rigoroso disporre di una trattazione che le comprendesse entrambe. Ma se si osserva che le rispettive bande di dispersione cadano a frequenze assai lontane, che le variazioni apportate all'indice di rifrazione dell'aria dalla presenza delle goccioline d'acqua sono minime, si riconosce che la rinuncia alla semplicità dei risultati da noi precedentemente esposti potrebbe non essere compensata, ai fini pratici, dai vantaggi della maggior generalità dei risultati ottenibili con una teoria unitaria.

Se ammettiamo la effettiva indipendenza degli effetti meccanico e termodinamico, le eccedenze dall'unità dei relativi indici di rifrazione vengono praticamente a sommarsi come si sommano $i$ coefficienti di assorbimento, cioè le quote di energia sonora assorbita in ogni tratto elementare del percorso dell'onda nella nube, per effetto della alterna condensazione del vapore d'acqua, e rispettivamente dell'attrito opposto dall'aria al movimento delle goccioline. Può considerarsi cioè un indice di rifrazione risultante dato approssimativamente da

$$
n-1 \cong v_{\mathrm{c}}+v_{\mathrm{a}}-j\left(u_{\mathrm{c}}+u_{\mathrm{a}}\right) .
$$

In corrispondenza ha luogo la considerazione del coefficiente di riflessione complessivo

$$
R=\left|\frac{n-1}{n+1}\right|^{2}
$$

che può costituirsi immediatamente richiamandosi alle espressioni 
[6] e [11] degli indici di rifrazione. Alfine di conseguire la massima semplicità dei risultati osserviamo però che, con approssimazione sempre sufficiente per lo scopo del nostro lavoro, possiamo nella precedente porre addirittura $|n+1|=2$, data l'esiguità degli scarti dei due indici di rifrazione dell'unità. Avremo allora approssimativamente

$$
R \supseteq \frac{1}{4}\left[\left(v_{\mathrm{c}}+v_{\mathrm{a}}\right)^{2}+\left(u_{\mathrm{c}}+u_{\mathrm{n}}\right)^{2}\right] .
$$

A parte la considerazione delle frequenze hasse in cui prevale nettamente l'effetto termodinamico, e delle alte frequenze in cui prevale invece quello meccanico, esiste una banda intermedia in cui $i$ due effetti sono dello stesso ordine: ciò succede allorché $u_{\mathrm{c}}$ diventa dello stesso ordine di $v_{a}$, nella quale circostanza, secondo i grafici di figg. 4 e $6, v_{a}$ è già trascurabile ed $u_{\mathrm{c}}$ ancora piccolo: in questa zona di passaggio il coefficente di riflessione è cioè dato approssimativamente da $\left[v^{2}+u^{2}{ }_{c}\right] / 4 \cong P_{c}+R_{a}$. Poiché poi al di fuori di questa banda uno dei due eoefficenti di riflessione è sempre piccolo rispetto all'altro possiamo ritenere che approssimativamente sia sempre

$$
R \cong R_{\mathrm{c}}+R_{\mathrm{a}} \text {. }
$$

Veniamo cosi ad ammettere che i due coefficienti di riflessione per condensazione e per attrito semplicemente si sommino in un unico coefficente di riflessione, che si mantiene all'incirca costante ed uguale ad $R_{\text {oc }}$ per le basse frequenze sino alla $\omega_{\mathrm{c}} / 2 \pi$, per diminuire poi rapidamente alle frequenze maggiori.

Esaminiamo ora come $\mathrm{i}$ risultati fin qui ottenuti si possano applicare alla riflessione della perturbazione sonora originata dal fulmine da parte degli ammassi nuvolosi. Se si considera la circostanza che la scarica del fulmine è generalmente un fenomeno aperiodico a fronte iniziale ripido, si può arguire che lo spettro delle frequenze sonore che ne origina debha essere costituito da componenti con ampiezze non molto diverse in un largo intervallo di frequenze; appare chiaro, secondo i risultati delle nostre considerazioni, come nell'incidenza su un banco di nubi possa residuare dopo la riflessione il solo gruppo delle frequenze più basse, sino al limite dell'una o dell'altra delle frequenze di taglio per condensazione o per attrito: non è agevole stabilire a priori quale di queste ultime giochi effettivamente a fissare l'estremo superiore della gamma riflessa, in quanto può darsi $i$ che la frequenza di taglio per condensazione, che secondo la [3] di- 
minuisce al diminuire di $q$ ed al crescere di $a$, venga a cadere al disotto della frequenza limite inferiore di udibilità per l'orecchio umano; questo succede normalmente per bassi contenuti di acqua nella nube, $q=10^{-7}$, e spesso anche per contenuti maggiori se le goccie sono grosse e rade. In questo caso rimane attiva la sola riflessione per attrito, la cui frequenza di taglio cresce pure al diminuire di $a$, essendo il minor valore del coefficiente di riflessione per attrito almeno in parte compensato dal rapido aumento della sensibilità dell'orecchio umano al crescere della frequenza, nel campo dei suoni bassi.

L'effetto della riflessione si compendia dunque nel fatto di essere conservate le sole componenti della perturbazione sonora più basse, e questo effetto si esalta ulteriormente se invece di una sola trattasi di più riflessioni successive: ciò conferisce al rombo del tuono un timbro sempre più cupo via via che lo stesso si prolunga; ed inoltre rende ragione della persistenza, spesso notevole, del rumore del fulmine, purché si tengano presenti la bassa velocità del suono nell'atmosfera e le relativamente grandi distanze da superare dall'onda sonora tra due riflessioni successive.

L'esiguità dei coefficienti di riflessione come risultano dal nostro calcolo non è di ostacolo alla interpretazione del fenomeno naturale, se si riflette alle due circostanze che si presentano congiunte, che la potenza sonora messa in gioco dallo scoppio del fulmine ̀̀ relativamente grande, e che la caratteristica di funzionamento dell'orecchio umano determina le sensazioni proporzionalmente al logaritmo del rapporto fra le potenze sonore ricevute: in effetti un coefficiente di riflessione $10^{-3}$ o $10^{-6}$, cioè dell'ordine di quello che risulta per la condensazione o rispettivamente per l'attrito, verrebbe ad equivalere ad una sensazione solo tre o sei volte minore di quella relativa all'onda incidente. In altre parole l'elemento che nel fenomeno che qui consideriamo fa sentire il suo peso non è la grandezza del coefficiente di riflessione per se stessa, ma piuttosto il suo andamento in funzione della frequenza: perché, con la soppressione nell'onda riflessa delle quote di energia corrispondenti alle componenti di frequenza elevata, residuano attive e, per quanto piccole, sufficienti all'audizione auricolare, le quote delle componenti di frequenza più bassa, che cadono al disotto della frequenza di taglio.

Un apprezzamento quantitativo dell'importanza di tutti i vari fattori che partecipano al fenomeno è ovviamente impossibile se si considera che altre circostanze entrano ancora a complicarlo, che le formazioni nuvolose sono lungi dal presentarsi sempre come lo strato 
piano da noi considerato, e che intervengono i già citati fatti della inomogeneità dell'atmosfera e del susseguirsi delle scariche elettriche.

In definitiva gli elementi di fatto che risultano dalle considerazioni teoriche da noi esposte. sono i seguenti : induhhiamente le componenti più basse della perturbazione sonora originata dal fulmine sono soggette ad una riflessione prevalente da parte delle nubi; se la riflessione è ripetuta si origina il caratteristico rimbombo del tuono, che può persistere a causa della bassa velocità del suono e della distanza delle nubi; ad ogni riflessione successiva le frequenze più basse vengono attenuate in misura minore di quelle più elevate, cioè il rimbombo tende a farsi sempre più cupo, in accordo con l'antica osservazione citata da Arago.

Roma - Istituto Nazionale di Geofisica - Giugno 1951.

\section{RIASSUNTO}

Si interpretano le cause fisiche di due fra le più singolari caratteristiche del rombo del tuono, e cioè la sua lunga persistenza ed il timbro eminentemente ricco di suoni bassi. Per quanto altri autori avessero in passato localizzato l'origine di questi fatti in svariate ed occasionali circostanze di ambiente, si è potuto dimostrare teoricamente nel presente lavoro come gli ammassi di goccioline di acqua che costituiscono le formazioni nuvolose esercitino una riflessione selettiva sulle onde sonore incidenti: precisamente tale riflessione avviene in misura apprezzabile solo per le componenti della perturbazione sonora, originata dallo scoppio del fulmine, di frequenza inferiore a certe due frequenze di taglio, proprie, l'una del fenomeno di alterna condensazione ed evaporazione delle goccioline sotto l'azione delle variazioni di pressione sonora, e l'altra del parziale trascinamento delle goccioline stesse nel moto dell'onda sonora per effetto della viscosità dell'aria. In base a questi risultati e tenuta anche presente la eventualità di più riflessioni successive si giustifica come, a causa della bassa velocità del suono nell'atmosfera e della relativamente grande distanza delle nubi, il rumore del tuono possa persistere a lungo; si può inoltre prevedere che il timbro sonoro debba farsi sempre più cupo, via via che il rimbombo si prolunga. 


\section{BIBLIOGRAFIA}

(1) Mac Graw - Hill ed. New York 1929, pag. 365.

(2) Meteor. ZS 3, 33, 1886.

(3) Journ. Acoust. Am. Soc. 21, 411, 1949.

(4) Oeuvres, pagg. 77 e 241, Baudry ed. Paris, 1854.

(5) Phys. ZS 42, 365, 1941.

(6) Ann. di Geofisica 3, 289, 1950.

(7) Cfr. loc. cit. a nota (6). 